\title{
THE DIPLAZONTINAE (HYMENOPTERA, ICHNEUMONIDAE) IN THE UKRAINIAN CARPATHIANS
}

\section{O. Varga}

Schmalhausen Institute of Zoology, NAS of Ukraine

vul. B. Khmelnytskogo, 15, Kyiv, 01030 Ukraine

E-mail: Sancho.Varga@gmail.com

O. Varga (https://orcid.org/0000-0002-6285-7830)

The Diplazontinae (Hymenoptera, Ichneumonidae) in the Ukrainian Carpathians. Varga, O. The Carpathian species of the subfamily Diplazontinae are reviewed. The list contains 48 species, 41 of which are reliably recorded from Ukraine for the first time. Species composition and high-altitude zone distribution of diplazontines in the studied region are discussed.

Key words: Hymenoptera, Ichneumonidae, Diplozotinae, new records, taxonomy, Ukraine, Carprtians.

\section{Introduction}

The subfamily Diplazontinae is a well defined group of pimpliform ichneumonids with 94 currently described species in the Western Palaearctic (Klopfstein, 2014; Vas, 2016), of which only 14 species reported from Ukraine so far (Besser, 1835; Meyer, 1936; Manukyan, 1988; Klopfstein, 2014).

The members of the subfamily Diplazontinae (except the genus Episemura) can be easily recognized by having tridentate mandible, rectangular, box-like first tergite, comparatively short propodeum, short ovipositor, and transverse head (Klopfstein 2014). The Western Palaearctic species were recently revised by Klopfstein (2014). In this work, she reported six species from Ukraine, mainly from the eastern part of the country: Diplazon laetatorius (Fabricius, 1781), Homotropus nigritarsus (Gravenhorst, 1829), H. pectoralis (Provancher, 1874), Promethes sulcator (Gravenhorst, 1829), Sussaba roberti Klopfstein, 2014, and Syrphophilus bizonarius (Gravenhorst, 1829). Nevertheless, the Ukrainian fauna of the subfamily Diplazontinae is still hugely underestimated. The first Ukrainian diplazontine record was Diplazon laetatorius provided by Besser (1835). Meyer (1936) reported six species: Bioblapsis polita (Vollenhoven, 1878), Campocraspedon caudatus (Thomson, 1890), Daschia brevitarsis (Thomson, 1890), Diplazon pectoratorius (Thunberg, 1822), Promethes nigriventris (Thomson, 1890), and P. sulcator, of which only the last one were found in Ukraine by later authors (Klopfstein, 2014). Unfortunately, the data remained questionable as the main collection of Meyer was lost. The Kasparyan's book chapter (1981) concerning diplazontines contained only key to genera and some notes on the general species richness in USSR. The first reliable record of the subfamily in Ukraine can be found in a paper of Manykyan (1988). He recorded three species from Ukraine, mainly from Crimea: Sussaba cognata (Holmgren, 
1858), S. erigator (Fabricius, 1793), and S. flavipes (Lucas, 1849). Thus, only nine diplazontine species can be considered as valid Ukrainian records before the present study.

All reliable host records of diplazontines refer to hoverfly family Syrphidae (Diptera). Most of the species are known as endoparasitoids of aphidophagous Syrphinae larvae. Only two species of the genus Bioblapsis have been reared from Eristalinae hosts feeding on fungus or tree sap (Rotheray, 1981, 1984, 1990).

\section{Material and methods}

The specimens examined for this study were collected by the author in various locations of the Ukrainian Carpathians and adjacent territories in 2009-2019. The material collected by the other entomologists and deposited in the collection of the I. I. Schmalhausen Institute of Zoology, Kyiv, Ukraine (SIZK) was also studied. The most common collecting sites of the Ukrainian Carpathians and respective abbreviations are reported below:

Ivano-Frankivsk Region (IF):

Mochary - Bogorodchany District, Mochary, $5 \mathrm{~km}$ NE of Bogorodchany, $315 \mathrm{~m}$, mixed forest (48.837078 N, 24.581379 E).

Dibrova - Bogorodchany District, Dibrova, $5 \mathrm{~km} \mathrm{SW}$ of Bogorodchany, $310 \mathrm{~m}$, oak forest (48.772054 N, 24.511657 E).

Zhbyr - Bogorodchany District, Zhbyr, $8 \mathrm{~km} \mathrm{SW}$ of Bogorodchany, $405 \mathrm{~m}$, mixed forest (48.777922 N, $24.456837 \mathrm{E})$.

Gorgany 1 - Bogorodchany District, Gorgany, 4 km SW of Stara Guta, 1050 m, coniferous forest (48.614163 N, 24.157944 E).

Gorgany 2 - Bogorodchany District, Gorgany, m. Syvulya, 12-13 km SW of Stara Guta, 1425 m, subalpine zone (48.531433 N, 24.132609 E).

Gorgany 3 - Bogorodchany District, Gorgany, m. Igrovets, 7 km SW of Stara Guta, 1375 m, border between coniferous forest and subalpine zone (48.598571 N, 24.130344 E).

Gorgany 4 - Bogorodchany District, Gorgany, m. Igrovets, 7.5 km SW of Stara Guta, 1500 m, subalpine zone (48.598571 N, 24.130344 E).

Elmy - Nadvirna District, Gorgany, Elmy, $15 \mathrm{~km} \mathrm{SW}$ of Yaremche, $950 \mathrm{~m}$, coniferous forest (48.415462 N, 24.418878 E).

Yavirnyk - Nadvirna District, Gorgany, m. Yavirnyk, 4-5 km SW of Yaremche, 1425 m, coniferous forest (48.407306 N, 24.498787 E).

Transcarpathian Region (ZAK):

Chorna Gora - Vynogradiv District, Chorna Gora, 280 m, oak forest (48.138338 N, 23.073689 E).

Kvasy - Rakhiv District, Svydovets, 2-3 km NW of Kvasy, 750 m, beech forest (48.144694 N, 24.270809 E).

Svydovets - Rakhiv District, Svydovets, 8 km NW of Kvasy, 1550 m subalpine zone (48.203921 N, 24.257559 E). Sheshul - Rakhiv District, Chornogora, Sheshul, 6-7 km NE of Kvasy, 1450 m, subalpine zone (48.157193 N, 24.363658 E).

Bilyi - Rakhiv District, Chornogora, Bilyi, coniferous forest (altitude different).

Marmarosy - Rakhiv District, Marmarosy, 11-12 km SE of Dilove, 1550 m, subalpine zone (47.925407 N, 24.311800 E).

Rakhiv - Rakhiv District, Rakhiv, 800-900 m, beech forest.

Kuziy - Rakhiv District, Kuziy, 450-700 m, beech forest.

Ugolka - Tyachiv District, 6.5 km N of Mala Ugolka, 600 m, beech forest (48.259464 N, 23.619672 E).

The specimens were collected mainly by sweep netting. If other method was used, e. g. Malaise trapping (MT), than it is metioned in the "Material examined" section. The species identification and general distribution follow Klopfstein (2014). Most of the specimens were identified to species, with the exception of most of Homotropus males sorted only to species groups and several Woldstedtius, Enizemum and Diplazon specimens sorted to morphospecies (which may possible represent new species or intraspecific variations). Photographs of specimens were taken with a Leica Z16 APO microscope equipped with Leica DFC 450 camera and processed by LAS Core soft ware (at SIZK) The high-altitude zones of the Ukrainian Carpathians follow Popov (1949).

\section{Results and discussion}

Species composition and high-altitude zone distribution

As a result of extensive field investigation of ichneumonid parasitoids (Hymenoptera, Ichneumonidae) in the Ukrainian Carpathians during last ten years more than 50 Diplazontinae morphospecies were collected. Most of them (48) can be identified 
using available key (Klopfstein, 2014), while the remaining specimens are probably refer to undescribed species. The additional material, including "fresh" material suitable for molecular analyses, is needed to solve this taxonomic problem. The aim of the present paper is to provide an overview of Diplazontinae species composition in the Ukrainian Carpathians.

The Carpathian diplazontines are represented by twelve genera, of which Enizemum, Phthorima, Syrphoctonus, Tymmophorus, and Woldstedtius were not previously reported from Ukraine. Four European genera, Episemura, Eurytyloides, Fossatyloides, and Xestopelta, remain unrecorded from the country. In general, the Ukrainian Carpathian diplazontines are well-represented, containing approximately the same number of species as neighboring countries of the Carpathian region, and at least a half of the European species (except Enizemum, Phthorima and Tymmophorus) for each genus (table 1).

Members of the subfamily Diplazontinae are represented in all high-altitude zones of the Ukrainian Carpathians (table 2). Oak foothill forest zone houses the largest number of species (34), seven of them found only in this zone. Among them, Woldtedtius merkli Vas, 2016 and Campocraspedon annulitarsis (Hedwig, 1938) were collected only in Precarpathian lowland, while Homotropus crassicornis Thomson, 1890 and Diplazon parvus Klopfstein, 2014 in more dry and hot lowland forests of Transcarpathia. Bioblapsis polita was found in both types of oak forests. In contrast to other pimpliform parasitoid groups (Varga, $2014 \mathrm{a}$, b, 2015, 2018) diplazontines are well-represented in the beech forest zone, containing almost the same number (32) of species as the oak forest zone. Four species, Diplazon pallicoxa Manukyan, 1987, Homotropus pallipes (Gravenhorst, 1829), Syrphoctonus idari Diller, 1985, and Syrphophilus scabriculus (Holmgren, 1858) have been found only in montane beech forests of Transcarpathia. The coniferous forest zone houses 28 species, of which two Diplazon species, D. flixi Klopfstein, 2014 and D. zetteli Klopfstein, 2014, and two Homotropus species, H. dimidiatus (Schrank, 1802) and H. megaspis (Thomson, 1890) are present only in high altitude coniferous forests of Chornogora. Another species, Promethes melanaspis (Thomson, 1890), was found only in Gorgany forests. Tweny-one species of Diplazontinae were collected on highest altitudes in the subalpine and alpine zones of Carpathians. Homotropus nigritarsus (Gravenhorst, 1829) is a specific species for this zone. Fourteen common and widely distributed species, Diplazon laetatorius, D. pectoratorius,

Table 1. Number of the Diplazontinae morphospecies found in the Ukrainian Carpathians compared to recorded species in the neighboring countries of the Carpathian basin and Europe ${ }^{\star}$

\begin{tabular}{|c|c|c|c|c|c|c|}
\hline Genus & $\begin{array}{c}\text { Ukrainian } \\
\text { Carpathians }\end{array}$ & Romania & Hungary & $\begin{array}{c}\text { Former } \\
\text { Czechoslovakia } \\
\end{array}$ & Poland & Europe \\
\hline Bioblapsis & 1 & & 1 & 1 & 1 & 2 \\
\hline Campocraspedon & 1 & 1 & 1 & 1 & 2 & 2 \\
\hline Daschia & & 1 & 1 & & 1 & 1 \\
\hline Diplazon & $12-13$ & 4 & 10 & 9 & 10 & 20 \\
\hline Enizemum & 2 & 1 & 1 & 2 & 3 & 5 \\
\hline Fossatyloides & & & & 1 & 1 & 1 \\
\hline Homotropus & $12-13$ & 7 & 11 & 12 & 17 & 21 \\
\hline Phthorima & 1 & 1 & 2 & 1 & 4 & 4 \\
\hline Promethes & 2 & 2 & 4 & 3 & 3 & 5 \\
\hline Sussaba & 7 & 7 & 5 & 5 & 6 & 11 \\
\hline Syrphoctonus & 4 & 2 & 3 & 3 & 1 & 5 \\
\hline Syrphophylus & 3 & 2 & 2 & 1 & 2 & 5 \\
\hline Tymmophorus & 1 & & 3 & 2 & 2 & 4 \\
\hline Woldstedtius & $4-7$ & 3 & 4 & 3 & 4 & 8 \\
\hline Xestopelta & & & 1 & 1 & 2 & 2 \\
\hline
\end{tabular}

*after Yu et al., 2012; Klopfstein, 2014; Vas, 2016. 
D. scutatorius Teunissen, 1943, Enizemum ornatum (Gravenhorst, 1829), Homotropus pictus (Gravenhorst, 1829), H. signatus (Gravenhorst, 1829), Promethes sulcator, Sussaba pulchella (Holmgren, 1858), Syrphoctonus tarsatorius (Panzer, 1809), Syrphophilus bizonarius (Gravenhorst, 1829), S. tricinctorius (Thunberg, 1822), Woldstedtius biguttatus (Gravenhorst, 1829), W. citropectoralis (Schmiedeknecht, 1926), and W. flavolineatus (Gravenhorst, 1829) were collected in all the high-altitude zones.

Table 2. High-altitude zone distribution of Diplazontinae in the Ukrainian Carpathians

\begin{tabular}{|c|c|c|c|c|}
\hline Species & Oak forest & Beech forest & Coniferous forest & Subalpine and alpine \\
\hline Bioblapsis polita & + & & & \\
\hline Campocraspedon annulitarsis & + & & & \\
\hline Diplazon angustus & & + & & + \\
\hline D. flixi & & & + & \\
\hline D. laetatorius & + & + & + & + \\
\hline D. neoalpinus & + & & & + \\
\hline D. pallicoxa & & + & & \\
\hline D. parvus & + & & & \\
\hline D. pectoratorius & + & + & + & + \\
\hline D. scutatorius & + & + & + & + \\
\hline D. tetragonus & + & + & + & \\
\hline D. tibiatorius & + & + & & \\
\hline D. varicoxa & + & + & & \\
\hline D. zetteli & & & + & \\
\hline Enizemum ornatum & + & + & + & + \\
\hline Homotropus collinus & + & & & + \\
\hline H. crassicornis & + & & & \\
\hline H. dimidiatus & & & + & \\
\hline H. elegans & + & & + & + \\
\hline H. frontorius & + & + & & \\
\hline H. haemorrhoidalis & + & & & \\
\hline H. longiventris & + & + & & \\
\hline H. megaspis & & & + & \\
\hline H. nigritarsus & & & & + \\
\hline H. pallipes & & + & & \\
\hline H. pictus & + & + & + & + \\
\hline H. signatus & + & + & + & + \\
\hline Phthorima compressa & + & + & & \\
\hline Promethes melanaspis & & & + & \\
\hline P. sulcator & + & + & + & + \\
\hline Sussaba cognata & + & + & & \\
\hline S. dorsalis & & + & + & \\
\hline S. erigator & + & + & + & \\
\hline S. flavipes & + & + & + & \\
\hline S. placita & & + & + & \\
\hline S. pulchella & + & + & + & + \\
\hline S. punctiventris & & & + & + \\
\hline Syrphoctonus fissorius & + & + & + & \\
\hline S. idari & & + & & \\
\hline S. tarsatorius & + & + & + & + \\
\hline Syrphophilus bizonarius & + & + & + & + \\
\hline S. scabriculus & & + & & \\
\hline S. tricinctorius & + & + & + & + \\
\hline Tymmophorus obscuripes & & + & + & + \\
\hline Woldstedtius biguttatus & + & + & + & + \\
\hline W. citropectoralis & + & + & + & + \\
\hline W. flavolineatus & + & + & + & + \\
\hline W. merkli & + & & & \\
\hline
\end{tabular}


Undetermined taxa

A single female of Diplazon tetragonus (Thunberg, 1822) collected in Chorna Gora had metasomal tergites 2 and 3 almost impunctate (punctures disappearing against the strongly coriaceous background sculpture which extends across transverse impressions onto hind part of the tergites). This character well matches with D. nordicus Klopfstein, 2014, which is closely related to $D$. tetragonus. This specimen remains under question until additional material from this locality will be collected.

One female of Enizemum collected in the coniferous forests of Chornogora has antenna black and with 24 flagellomeres. The specimen has median dorsal carinae on first tergite very long, almost reaching posterior margin, but evenly and roundedly converging towards each other. It dispays an intermediate condition of character between E. scutellare (Lange, 1911) and E. nigricorne (Thomson, 1890) and can not be reliably placed in any couplet of the key of Klopfstein (2014).

The genus Woldstedtius in the Carpathians probably contains several undescribed species. One female specimen collected in Mochary has 24 flagellomeres as in W. holarcticus (Diller, 1969), but differs in having mesoscutum and mid coxae entirely black ( W. holarcticus has yellow shoulder marks on mesoscutum and yellow stripe on mid coxae). In addition, this specimen is relatively large and morphologically similar to $W$. merkli. Thus, it is possible, that the coloration of coxae in $W$. merkli is variable. There are also three morphospecies similar to $W$. bauri Klopfstein, 2014 in having the antenna with at most 23 flagellomeres. One morphospecies has black mesoscutum (as W. bauri), but has entirely black mid coxae (black basally with yellow (reddish) apex in W. bauri). Another morphospecies has yellow stripe on mid coxae (as in W. holarcticus) and large yellow shoulder marks on mesoscutum. Both morphospecies were collected only in the subalpine zone in the same locality, but in different years. Several specimens of the third morphospecies were collected in lowland oak forest of Transcarpathia (Chorna Gora locality) and have mid coxae black basally and yellow with red apically (as in W. bauri), but have large yellow shoulder marks on mesosctutum and hind coxae largely red (black in W. bauri). Unfortunately, without additional material and molecular analyses it is impossible to ascertain if they represent distinct species or the coloration of mesoscutum and coxae is a variable character in species of the genus and can not be used for species identification. In any case, a new key to Woldstedtius species seems needed.

\section{List of species}

Bioblapsis polita (Vollenhoven, 1878) (fig. 1, A)

Material examined. Ukraine: IF: Mochary, MT, 10-31.08.2015, 1 ơ; ZAK: Chorna Gora, MT, 17.0510.06.2017, 1 ○; 2-22.07.2017, 1 ○; 20.05-7.06.2018, 1 \% (O. Varga).

Distribution. Palaearctic; first record for Ukraine.

Campocraspedon annulitarsis (Hedwig, 1938) (fig. 1, B)

Material examined. Ukraine: IF: Mochary, 3.05.2011, 3 ơ, 1 ○ (O. Varga).

Distribution. Western Palaearctic; first record for Ukraine.

\section{Diplazon angustus Dasch, 1964}

Material examined. Ukraine: ZAK: Sheshul, 16-18.06.2012, 5 o; 15.06.2013, 1 ơ, 4 ᄋ; 6.06.2014, 2 ᄋ; 26.06.2019, 1 \&; Chornogora, Dzhordzheva Preluka, 3 km NE of Kvasy, 48.173893 N, 24.311608 E, 1050 m, beech forest, 15.06.2012, 1 \% (O. Varga). 
Distribution. Holarctic; first record for Ukraine.

Remark. The Carpathian specimens have small yellow shoulder marks. Specimens collected in the same locality show variability in punctation of face, from densely punctate to almost impunctate.

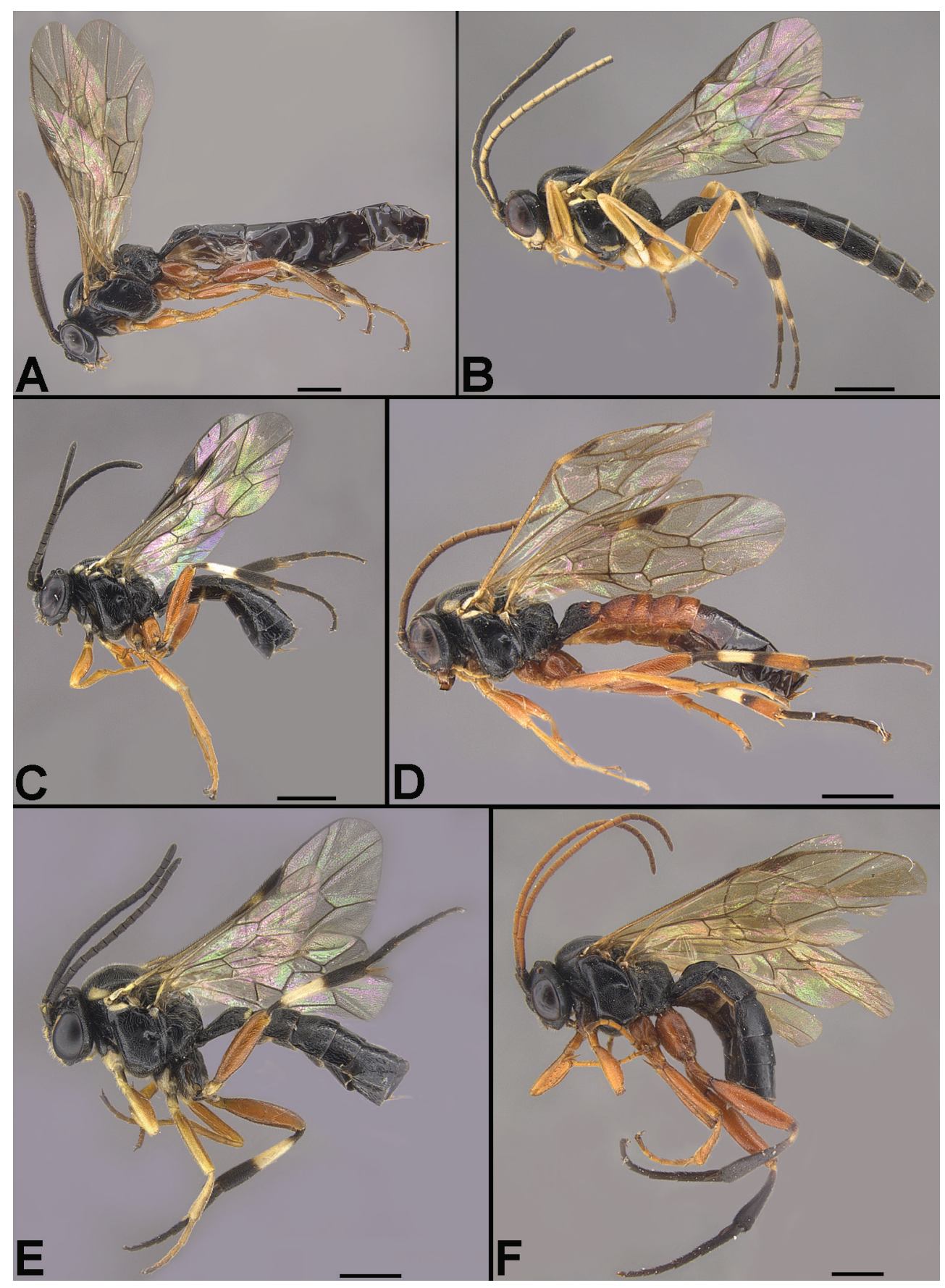

Fig. 1. The Carpathian Diplazontinae, habitus (lateral view). A - Bioblapsis polita, female; B - Campocraspedon annulitarsis, male; $\mathrm{C}-$ Diplazon parvus, female; D - D. laetatorius, female; $\mathrm{E}-\mathrm{D}$. varicoxa, female; $\mathrm{F}-$ Enizemum ornatum, female. Scale bar $=1 \mathrm{~mm}$. 
Diplazon flixi Klopfstein, 2014

Material examined.Ukraine: ZAK: Bilyi, 1000-1200 m, 8.08.1994, 1@ (A. Kotenko).

Distribution. Western Palaearctic; first record for Ukraine.

Diplazon laetatorius (Fabricius, 1781) (fig. 1, D)

Material examined. Ukraine: IF: Mochary, 25.05.2010, 1 \%; 27.05.2010, 1 \%; 28.06.2010, 1 \%; 30.08.2012, 1 ᄋ; MT, 16.06-4.07.2014, 1 \}; 3.08-14.09.2014, 1 ○; 14-30.06.2015, 1 ○; 30.06-21.07.2015, 9 ᄋ; 21.07-10.08.2015, 1 ○; 31.08-22.09.2015, 3 ○; 5.07-1.08.2018, 1 ○; Gorgany 1, 1.07.2012, 1 o; Gorgany 3, MT, 2.07-8.08.2014, 2 o; Tysmenytsya Distr., Ivano-Frankivsk, 18.05.2009, 1 o (O. Varga); Rybne, 48.939894 N, 24.582188 E, MT, 26.05-16.06.2019, 1 ᄋ (V. Shparyk); Nadvirna Distr., Kukul, 30.06.1975, 2 ○; 24.07.1972, 1 ९; 26.07.1972, 1 \%; Chornogora, m. Pozhezhevska, 19.07.1972, 7 \%; 20.07.1972, 2 o; Chornogora, m. Dantsizh, 20.07.1972, 2 ○ (V. Tolkanitz); ZAK: Beregove Distr., Bene, oak forest, 48.164673 N, 22.772055 E, MT, 10.06-2.07.2017, 1 ○; MT, 2-22.07.2017, 1 \%; Chorna Gora, MT, 5-23.06.2016, 3 ○; 17.05-10.06.2017, 1 ○; 10.06-2.07.2017, 3 \%; 2-22.07.2017, 2 \%; 12.04-2.05.2018, 2 ○; 20.05-7.06.2018, 1 ○; 7.06-3.07.2018, 3 ᄋ; 3-26.07.2018, 1 ○; 26.07-18.08.2018, 1 ᄋ (O. Varga); 5.06.1969, 1 ᄋ (V. Tolkanitz); Kvasy, 7.07.2012; 1 ᄋ; MT, 24.06-14.07.2013, 1 ○; 7.05-5.06.2014, 1 ○; 5-29.06.2014, 1 ○; 29.06-15.07.2014, 2 ᄋ; 10.08-1.09.2014, 2 ᄋ; Sheshul, 9.07.2011, 1 ○; MT, 5-29.06.2014, 2 ○; 29.06-18.07.2014, 2 ○; Marmarosy, 29.07.2013, 2 ○ (O. Varga); ? Marmarosy, Kudriavyi, 10.07.1969, 3 ○; 11.07.1969, 3 o; Rakhiv, 29.07.1994, 2 o; 31.07.1994, 1 o; 1.08.1994, 3 o; 4.08.1994, 1 ф; Kuziy, 12.08.1994, 1 ᄋ (A. Kotenko); Dilove, 07.2008, 1 ○; 15.06.2009, 1 ᄋ (R. Bidychak); Bogdan, Golovach, 29.07.1972, 1 ○; Svalyava Distr., Polyana, 28.06.1986, 1 ○; abandoned orchard, 9.07.1986, 4 ○; 3 km W of Polyana, 12.07.1986, 7 o; Uklyn, 10.07.1986, 1 ○; Uzhgorod Distr., Uzhgorod, abandoned orchard, 3.07.1986, 1 \%; Khust Distr., Khust, 15.08.1963, 2 ९; 17.08.1963, 2 ○; 25.08.1963, 2 @; Lviv Region: Turka, 23.06.1969, 1 o (V. Tolkanitz).

Distribution. Cosmopolitan species; Ukraine (Klopfstein, 2014).

Diplazon neoalpinus Zwakhals, 1979

Material examined. Ukraine: IF: Dibrova, 2.06.2012, 1 \&; ZAK: Sheshul, 26.06.2019, 1 ९ (O. Varga).

Distribution. Holarctic; first record for Ukraine.

Diplazon pallicoxa Manukyan, 1987

Material examined. Ukraine: ZAK: Kuziy, 12.08.1994, 2 ơ, 2 ○ (A. Kotenko).

Distribution. Palaearctic; first record for Ukraine.

Diplazon parvus Klopfstein, 2014 (fig. 1, C)

Material examined.Ukraine: ZAK: Chorna Gora, MT, 20.05-7.06.2018, 2 o; 7.06-3.07.2018, 2 ᄋ; 3-26.07.2018, 1 \%; 26.07-18.08.2018, 1 \% (O. Varga).

Distribution. Palaearctic; first record for Ukraine.

\section{Diplazon pectoratorius (Thunberg, 1822)}

Material examined. Ukraine: IF: Mochary, 25.05.2010, 1 o; 10.08.2010, 1 \%; MT, 10-29.04.2014, 1 \%; Dibrova, 8.10.2013, 3 ○; Gorgany 1, 24.05.2014, 2 ơ; Gorgany 2, 14.07.2012, 1 o; Gorgany 3, 20.06.2013, 3 ᄋ; Yavirnyk, 14-16.08.2010, 1 ф (O. Varga); Chornogora, m. Goverla, 1500 m, 26.06.1975, 3 o; Chornogora, m. Pozhezhevska, 12.07.1979, 1 ф; 15.07.1979, 2 ○ (V. Ermolenko); ZAK: Beregove Distr., Beregove, 15.06.1963, 1 o; Chorna Gora, MT, 2-17.05.2017, 1 ᄋ; Kvasy, 5.06.2010, 1 o", 2 o; Svydovets, 27.09.2011, 2 ơ, 1 ᄋ; Sheshul, 9.07.2011, 2 ○; 16-18.06.2012, 1 ○; 15.06.2013, 2 ○, 5 ᄋ; 6.06.2014, 1 ○; MT, 5-29.06.2014, 1 ᄋ; 29.06-18.07.2014, 1 ㅇ (O. Varga); Bilyi, 1000-1200 m, 7.08.1994, 1 @; Rakhiv, 2.08.1994, 3 ㅇ (A. Kotenko); Yasynya, 15.07.1963, 1 ○ (V. Ermolenko); Uzhgorod Distr., Kamyanytsya, 4.10.1980, 7 ○’, 12 @ (A. Kotenko).

Distribution. Holarctic, Neotropical and Oriental Regions; first record for Ukraine. 
Diplazon scutatorius Teunissen, 1943

Material examined. Ukraine: IF: Mochary, MT, 3.08-14.09.2014, 1 ○; 30.06-21.07.2015, 1 ᄋ; Dibrova, 6.06.2013, 1 ф; Elmy, 14.07.2011, 1 ф; ZAK: Chorna Gora, MT, 9.09-2.10.2018, 1 ○; Kvasy, MT, 5-29.06.2014, 1 ф; Sheshul, 16-18.06.2012, 1 ф; Marmarosy, 6-9.08.2012, 1 ơ (O. Varga); Bilyi, 1000-1200 m, 5.08.1994, 1 ᄋ; Rakhiv, 31.07.1994, 2 ९ (A. Kotenko); Ugolka, MT, 12-31.05.2015, 1 ф (O. Varga).

Distribution. Palaearctic; first record for Ukraine.

Diplazon tetragonus (Thunberg, 1822)

Material examined. Ukraine: IF: Mochary, 27.06.2009, 1 o; Dibrova, 26.09.2010, 1 o; 8.10.2013, 1 ᄋ; 11.10.2013, 1 o; Bogorodchany, 10.05.2010, 1 o'; Zhbyr, 29.09.2012, 1 o'; ZAK: Chorna Gora, MT, 2-22.07.2017, 1 ○; (O. Varga); Bilyi, 1000-1200 m, 5.08.1994, 2 ९; 6.08.1994, 1 ○; Kuziy, 12.08.1994, 1 ơ, 2 @ (A. Kotenko).

Distribution. Holarctic and Oriental Regions; first record for Ukraine.

\section{Diplazon tibiatorius (Thunberg, 1822)}

Material examined. Ukraine: ZAK: Chorna Gora, MT, 17.05-10.06.2017, 1 ф; 10.06-2.07.2017, 1 ; Ugolka, MT, 22.05-8.06.2015, 1 \& (O. Varga).

Distribution. Holarctic and Neotropical Regions; first record for Ukraine.

Remarks. Smaller specimens collected in the same locality (Chorna Gora) are generally less strongly punctate on both mesopleuron and metasoma and have been identified as $D$. parvus. However, it is possible that they represent just a variation of $D$. tibiatorius.

Diplazon varicoxa (Thomson, 1890) (fig. 1, E)

Material examined. Ukraine: ZAK: Chorna Gora, MT, 20.05-7.06.2018, 8 ᄋ; 7.06-3.07.2018, 1 \%; Kvasy, MT, 14.07-24.08.2013, 1 क; 6.08-3.09.2015, 1 \& (O. Varga).

Distribution. Palaearctic and Oriental Regions; first record for Ukraine.

Diplazon zetteli Klopfstein, 2014

Material examined. Ukraine: IF: Nadvirna Distr., Chornogora, m. Pozhezhevska, 1000 m, 26.06.1975, 4 ơ, 2 ㅇ (V. Ermolenko).

Distribution. Palaearctic; first record for Ukraine.

Enizemum ornatum (Gravenhorst, 1829) (fig. 1, F)

Material examined. Ukraine: IF: Mochary, 17.05.2009, 1 ○; Dibrova, 8.10.2013, 2 ○; Gorgany 1, 13.07.2012, 1 \%; 18.07.2015, 1 ф; Gorgany 2, 14.07.2012, 1 ơ, 3 ९ (O. Varga); Nadvirna Distr., Chornogora, m. Pozhezhevska, 15.07.1979, 3 o (V. Ermolenko); ZAK: Sheshul, 29.06-18.07.2014, 2 ○ (O. Varga); 9.08.1994, 1 ○; Marmarosy, 11.08.1994, 2 ○ (A. Kotenko); 11.08.2009, 1 ○; Marmarosy, m. Mika-Mare, 30-31.07.2013, 1 ㅇ (O. Varga); Rakhiv, 2.08.1994, 3 ơ, 2 ᄋ; Chornogora, m. Goverla, 8.08.1994, 5 œ; Bilyi, 1400-1500 m, 5.08.1994, 2 ○; 6.08.1994, 1 ○; 7.08.1994, 24 o; 8.08.1994, 40 ᄋ; Uzhgorod Distr., Kamyanytsya, 4.10.1980, 1 ᄋ (A. Kotenko).

Distribution. Holarctic and Oriental Regions; first record for Ukraine.

\section{Homotropus collinus (Stelfox, 1941) (fig. 2, A)}

Material examined. Ukraine: IF: Gorgany 4, MT, 24.05-16.06.2014, 1 ○ (O. Varga); Tysmenytsya Distr., Rybne, 48.939894 N, 24.582188 E, MT, 26.05-16.06.2019, 1 ㅇ (V. Shparyk); ZAK: Sheshul, 6.06.2014, 1 ㅇ (O. Varga).

Distribution. Western Palaearctic; first record for Ukraine.

Remarks. The Carpathian specimens have coxae black with orange marks (as Homotropus melanogaster (Holmgren, 1872)). Only one specimen has very strongly excised clypeus. 
Homotropus crassicornis Thomson, 1890 (fig. 2, B)

Material examined. Ukraine: ZAK: Beregove Distr., Beregove, 26.05.1963, 1 \%; Chorna Gora, MT, 28.04-19.05.2016, 1 \%; 17.05-10.06.2017, 2ᄋ; 20.05-7.06.2018, 2 \% (O. Varga).

Distribution. Western Palaearctic; first record for Ukraine.

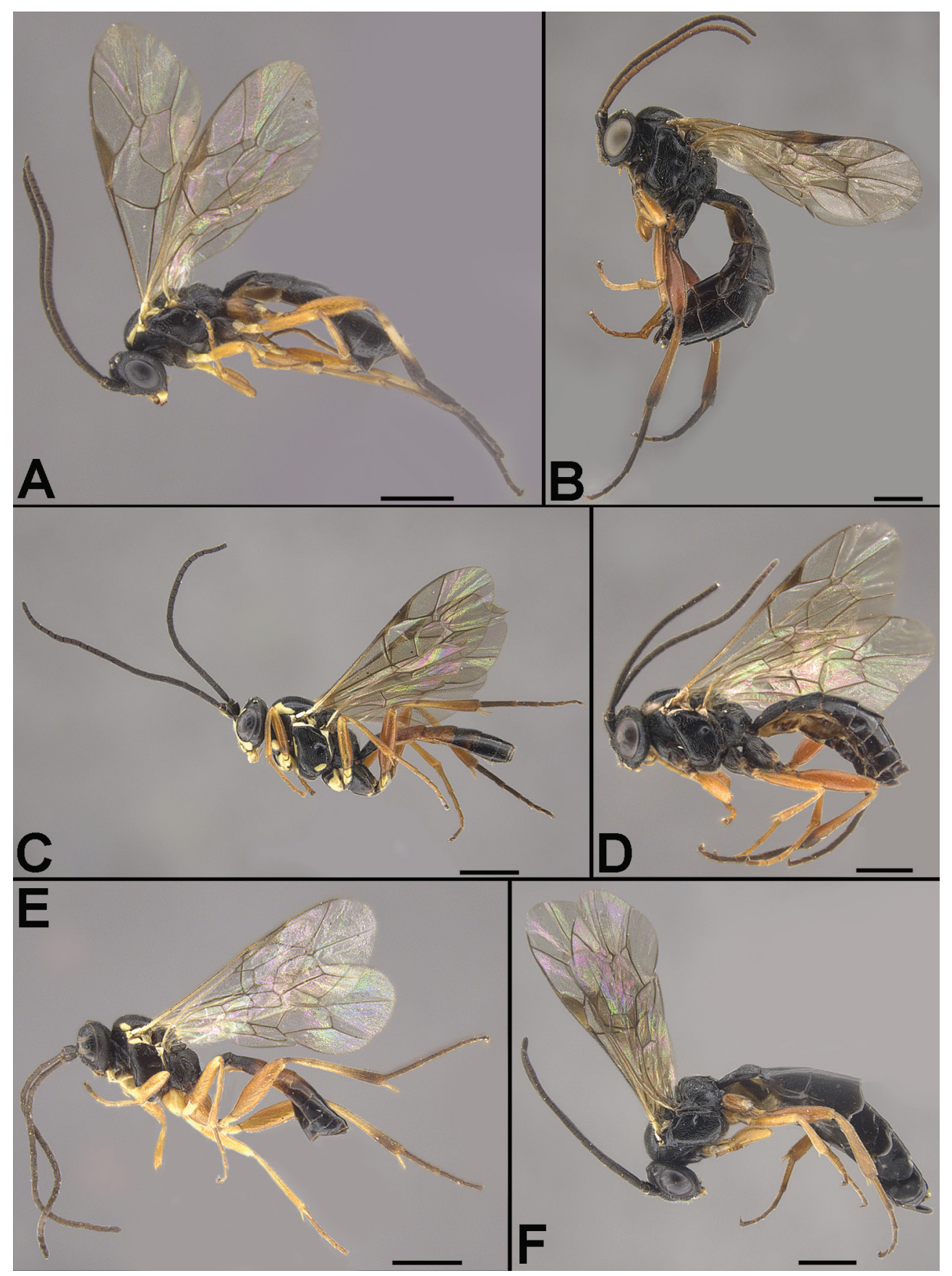

Fig. 2. The Carpathian Diplazontinae, habitus (lateral view). A - Homotropus collinus, female; B H. crassicornis, female; $\mathrm{C}-$ Homotropus elegans, male; D - H. nigritarsus, female; $\mathrm{E}-\mathrm{H}$. pallipes, female; $\mathrm{F}-$ Phthorima compressa, female. Scale bar $=1 \mathrm{~mm}$. 
Homotropus dimidiatus (Schrank, 1802)

Material examined. Ukraine: Ivano-Frankivsk Reg.: Nadvirna Distr., Chornogora, 1300-1400 m, 13.07.1979, 1 ᄋ (V. Ermolenko); ZAK: Bilyi, 1000-1200 m, 7.08.1994, 1 ㅇ (A. Kotenko).

Distribution. Holarctic and Oriental Regions; first record for Ukraine.

Homotropus elegans (Gravenhorst, 1829) (fig. 2, C)

Material examined. Ukraine: IF: Mochary, 17.05.2009, 1 o; 9.07.2009, 2 o; 29.07.2009, 1 O; 22.05.2010, 1 o', 1 ○; 23.05.2010, 1 o; 7.06.2010, 1 o'; 9.05.2011, 1 o; 3.09.2011, 3 o'; 24.04.2012, 1 o; 25.04.2012, 2 o'; 28.04.2012, 7 ơ; Dibrova, 26.05.2012, 1 o'; Zhbyr, 2.05.2012, 2 o'; Gorgany 1, 1.07.2012, 1 ơ; Gorgany 2, 14.07.2012, 1 ơ (O. Varga); Nadvirna Distr., Chornogora, m. Dantsizh, 20.07.1972, 1 \& (V. Tolkanitz); ZAK: Marmarosy, 1.08.1994, 1 \& (A. Kotenko).

Distribution. Holarctic; first record for Ukraine.

Homotropus frontorius (Thunberg, 1824)

Material examined. Ukraine: IF: Mochary, 3.05.2011, 1 क; ZAK: Kvasy, 4.05.2012, 1 \% (O. Varga).

Distribution. Holarctic; first record for Ukraine.

Homotropus haemorrhoidalis (Szépligeti, 1898)

Material examined. Ukraine: IF: Dibrova, 26.09.2010, 1 @ (O. Varga).

Distribution. Palaearctic; first record for Ukraine.

Homotropus longiventris Thomson, 1890

Material examined. Ukraine: ZAK: Chorna Gora, MT, 17.05-10.06.2017, 2 ९; Ugolka, MT, 6.083.09.2015, 1 ㅇ (O. Varga).

Distribution. Palaearctic; first record for Ukraine.

Homotropus megaspis (Thomson, 1890)

Material examined. Ukraine: IF: Gorgany 1, 8-9.06.2012, 1 ○ (O. Varga); ZAK: Bilyi, 1400-1500 m,

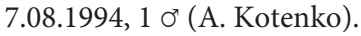

Distribution. Palaearctic; first record for Ukraine.

Homotropus nigritarsus (Gravenhorst, 1829) (fig. 2, D)

Material examined. Ukraine: ZAK: Sheshul, 5-29.06.2014, 1 ○; 18.07-10.08.2014, 2 ○; 26.06.2019, 1 o; Marmarosy, m. Mika-Mare, 30-31.07.2013, 1 o (O. Varga); Chornogora, m. Goverla, 8.08.1994, 2 ᄋ; (A. Kotenko).

Distribution. Holarctic and Neotropical Regions; Ukraine (Klopfstein, 2014).

Homotropus pallipes (Gravenhorst, 1829) (fig. 2, E)

Material examined. Ukraine: ZAK: Kvasy, 23.08.2009; 1 @ (O. Varga); Dilove, 07.2008, 1 ○; 0607.2009, 1 ○ (R. Bidychak).

Distribution. Holarctic and Neotropical Regions; first record for Ukraine.

\section{Homotropus pictus (Gravenhorst, 1829)}

Material examined. Ukraine: IF: Dibrova, 8.10.2013, 1 \%; Gorgany 2, 1700 m, 14.07.2012, 1 ; Gorgany 3, MT, 2-20.07.2014, 1 o; 20.07-8.08.2014, 1 ᄋ; 8-28.08.2014, 1 o; Gorgany 4, MT, 2-20.07.2014, 4 ९; 20.07-8.08.2014, 1 \%; 8-28.08.2014, 1 \%; ZAK: Sheshul, MT, 29.06-18.07.2014, 3 q (O. Varga); Bilyi, 10001200 m, 6.08.1994, 1 ○ (A. Kotenko); Ugolka, MT, 12-31.05.2015, 1 ф (O. Varga). 
Distribution. Palaearctic; first record for Ukraine.

Remarks. Several specimens have almost indistinct yellow face patch (from brownish to almost black). One female has yellow face patch confluent with clypeus. Some of the specimens have pterostigma dark with yellow line above, not pale centrally.

Homotropus signatus (Gravenhorst, 1829)

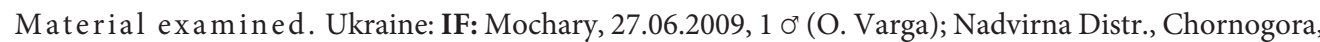
m. Pozhezhevska, 19.07.1972, 1 (V. Tolkanitz); ZAK: Rakhiv, 29.07.1994, 1 ○; 1.08.1994, 1 ○ (A. Kotenko); Bogdan, Golovach, 28.07.1972, 2 ○ (V. Tolkanitz); Khust Distr., Khust, 20.08.1963, 1 ‥

Distribution. Holarctic; first record for Ukraine.

Phthorima compressa (Desvignes, 1856) (fig. 2, F)

Material examined. Ukraine: ZAK: Chorna Gora, MT, 20.05-7.06.2018, 1 \%; Kvasy, 5.06.2010, 1 o (O. Varga); Dilove, 7.08.2008, 1 ơ (R. Bidychak).

Distribution. Holarctic; first record for Ukraine.

Promethes melanaspis (Thomson, 1890)

Material examined. Ukraine: IF: Gorgany 1, 1.07.2012, 1 ơ (O. Varga).

Distribution. Holarctic; first record for Ukraine.

Promethes sulcator (Gravenhorst, 1829) (fig. 3A)

Material examined. Ukraine: IF: Mochary, 17.05.2009, 1 ○; 3.08.2011, 1 o; 4.09.2011, 1 ○; MT, 21.07-10.08.2015, 1 o'; Zhbyr, 13.09.2011, 1 ○; Yavirnyk, 14-16.08.2010, 1 ○ (O. Varga); Chornogora, m. Dantsizh, 20.07.1972, 2 ơ (V. Tolkanitz); ZAK: Kvasy, 23.08.2009; 1 ○ (O. Varga); Sheshul, 23.07.1972, 1 ơ, 1 ᄋ (V. Tolkanitz); 9.08.1994, 2 ơ, 1 ᄋ (A. Kotenko); 29.06.2014, 1 ᄋ; MT, 29.06-18.07.2014, 2 ᄋ; 18.07-10.08.2014, 1 o'; 10.08-1.09.2009, 3 o; Marmarosy, 6-9.08.2012, 1 ᄋ (O. Varga); 11.07.1994, 1 o; ?Marmarosy, Kudriavyi, 10.07.1969, 3 \%; Rakhiv, 29.07.1994, 4 ơ, 5 ᄋ; 31.07.1994, 2 ơ, 2 \%; 1.08.1994, 1 \%; 2.08.1994, 4 @; Chornogora, m. Goverla, 8.08.1994, 2 o'; Bilyi, 1400-1500 m, 7.08.1994, 1 ơ; 8.08.1994, 3 ơ, 5 o (A. Kotenko); Bogdan, Golovach, 5.07.1969, 2 ○; 28.07.1972, 2 ○; Kosivska Polyana, 10.07.1969, 2 ○; Svalyava Distr., Polyana, abandoned orchard, 7.07.1986, 1 ơ, 1 ○; 3 km W of Polyana, 12.07.1986, 1 \& (V. Tolkanitz); Khust Distr., Khust, 20.08.1963, 2 ○; 26.08.1963, 1 ○.

Distribution. Holarctic and Oriental Regions; Ukraine (Klopfstein, 2014).

\section{Sussaba cognata (Holmgren, 1858) (fig. 3, B)}

Material examined. Ukraine: ZAK: Chorna Gora, MT, 22.07-12.08.2018, 1 @; Kvasy, MT, 6.083.09.2015, 1 ᄋ; Ugolka, MT, 8.07-6.08.2015, 2 ९; 6.08-3.09.2015, 1 ९ (O. Varga).

Distribution. Holarctic and Oriental Regions; Ukraine (Manukyan, 1988).

Sussaba dorsalis (Holmgren, 1858) (fig. 3, C)

Material examined. Ukraine: IF: Gorgany 1, 8.08.2011, 1 ○ (O. Varga); ZAK: Rakhiv Distr., Chornogora, m. Goverla, 8.08.1994, 1 \%; Bilyi, 1400-1500 m, 7.08.1994, 1 @ (A. Kotenko); Svalyava Distr., Polyana, abandoned orchard, 7.07.1986, 1 q (V. Tolkanitz).

\section{Distribution. Holarctic; first record for Ukraine.}

Sussaba erigator (Fabricius, 1793)

Material examined. Ukraine: IF: Mochary, 25.05.2010, 1 o'; 27.05.2010, 1 ơ; 12.06.2012, 1 ơ; MT, 14-30.06.2015, 1 ○; Dibrova, 14.06.2012, 1 ○; Tysmenytsya Distr., Ivano-Frankivsk, Vovchynets, 11.08.2010 1 ○; 11.09.2011 1 ᄋ (O. Varga); ZAK: Rakhiv, 1.08.1994, 1 o; Kuziy, 12.08.1994, 1 ơ, 1 ○ (A. Kotenko); Bogdan, Golovach, 29.07.1972, 1 q (V. Tolkanitz); Khust Distr., Khust, 25.08.1963, 1 0’.

Distribution. Palaearctic; Ukraine (Manukyan, 1988). 


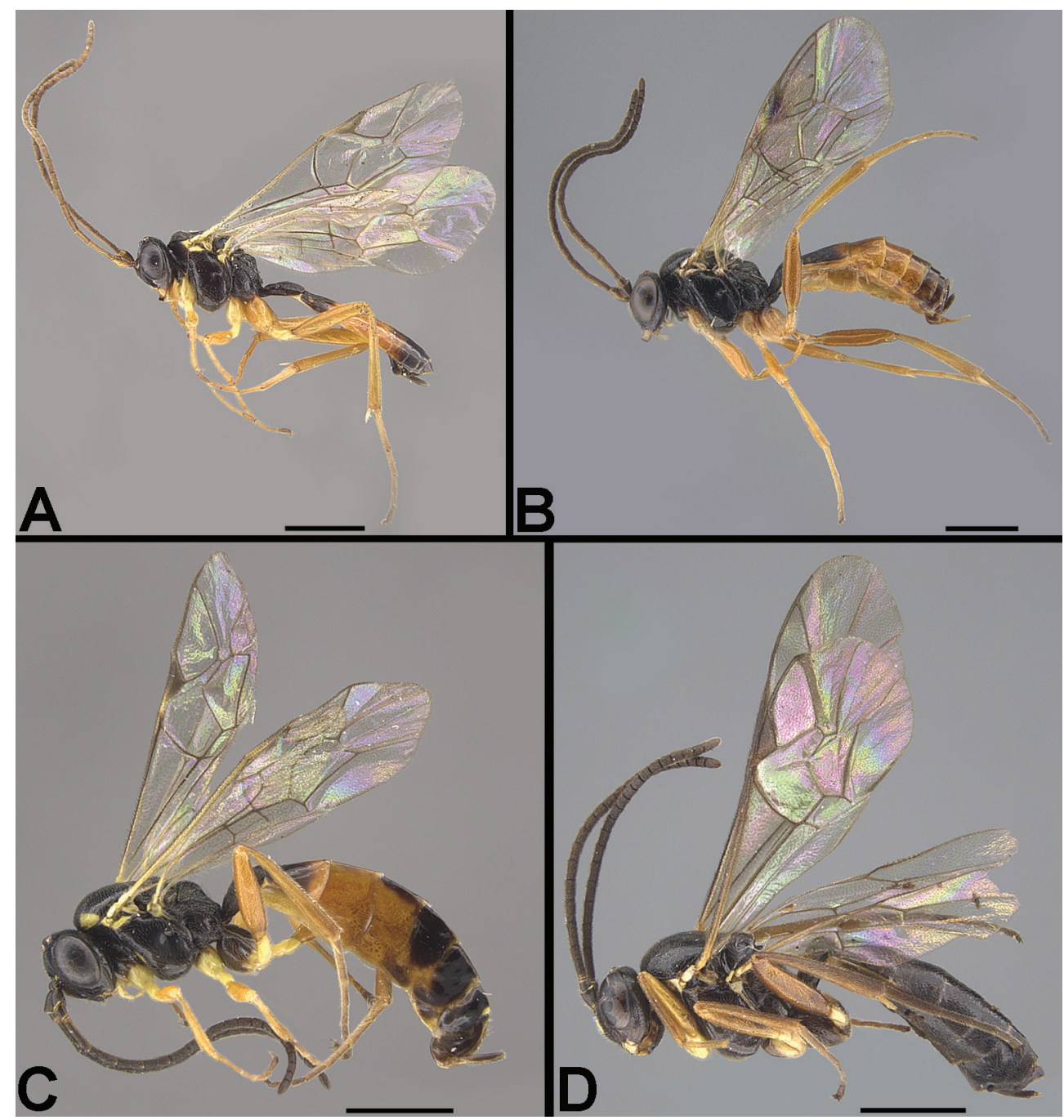

Fig. 3. The Carpathian Diplazontinae, female habitus (lateral view). A - Promethes sulcator; B - Sussaba cognata; C - S. dorsalis; D - S. punctiventris. Scale bar $=1 \mathrm{~mm}$.

\section{Sussaba flavipes (Lucas, 1849)}

Material examined. Ukraine: IF: Mochary, 17.05.2009, 2 o; 18-19.05.2012, 1 o; Dibrova, 8.05.2011, 2 ơ, 1 ९; Pidhirya, 24.07.2009, 1 ᄋ; Tysmenytsya Distr., Ivano-Frankivsk, Vovchynets, 11.09.2011, 1 ○" (O. Varga); ZAK: Kvasy, 23.08.2009, 1 o'; MT, 15.07-10.08.2014, 1 ○; 10.08-1.09.2014, 1 ○ (O. Varga); ? Marmarosy, Kudriavyi, 11.07.1969, 1 ○; Bilyi, 5.08.1994, 1 ᄋ; 6.08.1994, 1 ơ, 8.08.1994, 1 ơ; Rakhiv, 31.07.1994, 2 ○; 1.08.1994, 1 ○; 2.08.1994, 1 ○; Kuziy, 12.08.1994, 1 ơ, 3 ○ (A. Kotenko); Ugolka, yellow pan trap, 1718.07.2014, 1 ศ", 1 ᄋ (O. Varga).

Distribution. Holarctic; Ukraine (Manukyan, 1988).

\section{Sussaba placita Dasch, 1964}

Material examined. Ukraine: IF: ZAK: Rakhiv Distr., Chornogora, m. Goverla, 1500 m, 26.06.1975, 1 ९; Uzhgorod Distr., Kamyanytsya, 4.10.1980, 2 ○ (A. Kotenko).

Distribution. Western Palaearctic and Nearctic Regions; first record for Ukraine. 


\section{Sussaba pulchella (Holmgren, 1858)}

Material examined. Ukraine: IF: Mochary, MT, 31.08-22.09.2015, 1 ○; Gorgany 1, 1.07.2012, 1 \%; 18.07.2015, 1 o', 1 ᄋ; Gorgany 2, 14.07.2012, 6 ơ, 3 ᄋ; Nadvirna Distr., Chornogora, m. Goverla, 2060 m, 28.07.2012, 1 ơ (O. Varga); Kukul, 24.07.1972, 1 @; Chornogora, m. Pozhezhevska, 20.07.1972, 1 ○’, 2 ф; Chornogora, m. Dantsizh, 20.07.1972, 2 ơ 1 o (V. Tolkanitz); ZAK: Kvasy, MT, 15.07-10.08.2014, 1 o (O. Varga); Sheshul, 23.07.1972, 2 ơ, 1 ○ (V. Tolkanitz); 9.08.1994, 1 ○ (A. Kotenko); MT, 29.06-18.07.2014, 1 ○; 18.07-10.08.2014, 1 ○', 1 ᄋ; Marmarosy,

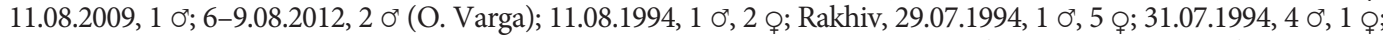
1.08.1994, 4 ○; 2.08.1994, 2 ○", 6 ○; 3.08.1994, 1 ○; 4.08.1994, 1 ○; 13.08.1994, 1 ○; Chornogora, m. Goverla, 8.08.1994, 1 ơ, 1 ○; Bilyi, 1400-1500 m, 5.08.1994, 2 ○; 6.08.1994, 1 o; 7.08.1994, 1 ơ, 3 o; 8.08.1994, 2 ơ, 2 o; Kuziy, 12.08.1994, 1 ○ (A. Kotenko); Bogdan, Golovach, 29.07.1972, 1 ९ (V. Tolkanitz); Ugolka, 16.05.2004, 1 ९ (R. Bihychak).

Distribution. Holarctic and Oriental Regions; first record for Ukraine.

\section{Sussaba punctiventris (Thomson, 1890) (fig. 3, D)}

Material examined. Ukraine: IF: Gorgany 2, 1700 m, 14.07.2012, 1 ơ, 2 ○; Gorgany 3, MT, 13.062.07.2014, 1 @ (O. Varga); Nadvirna Distr., Chornogora, 1300-1400 m, 13.07.1979, 1 ф (V. Ermolenko); ZAK: Sheshul, 26.06.2019, 1 ơ, 1 ᄋ; Chornogora, m. Petros, 1900-1950 m, 17.06.2012, 1 q (O. Varga); Chornogora, m. Goverla, 8.08.1994, 1 ○; Bilyi, 1400-1500 m, 8.08.1994, 1 ㅇ (A. Kotenko).

\section{Distribution. Western Palaearctic and Nearctic Regions; first record for Ukraine.}

\section{Syrphoctonus fissorius (Gravenhorst, 1829)}

Material examined. Ukraine: IF: Mochary, 27.05.2010, 1 ф; Dibrova, 26.09.2010, 2 ○; Zhbyr, 13.09.2011, 1 \&; Gorgany 1, 20-22.05.2012, 1 \%; Nadvirna Distr., 6 km E of Vorokhta, 16.07.1979, 1 ㅇ (O. Varga); Chornogora, 1300-1400 m, 13.07.1979, 3 (V. Tolkanitz); ZAK: Kvasy, 5.06.2010, 1 ○', 2 \%; Svydovets, 7.06.2012, 1 \% (O. Varga); Rakhiv, 31.07.1994, 3 \%; 2.08.1994, 1 đ’, 10 ९ (A. Kotenko).

\section{Distribution. Palaearctic; first record for Ukraine.}

\section{Syrphoctonus idari Diller, 1985}

Material examined. Ukraine: ZAK: Rakhiv, 2.08.1994, 1 ○ (A. Kotenko).

Distribution. Western Palaearctic; first record for Ukraine.

\section{Syrphoctonus tarsatorius (Panzer, 1809)}

Material examined. Ukraine: IF: Mochary, 29.04.2010, 1 ơ; 28.06.2010, 1 \%; 23.09.2010, 1 ○; MT, 10 29.04.2014, 1 ᄋ; Dibrova, 26.04.2009, 1 ơ; 8.10.2013, 3 ơ, 3 ᄋ; 14.06.2012, 1 o; Zhbyr, 29.09.2012, 1 ơ; Gorgany 3, 20.06.2013, 4 ○; Tysmenytsya Distr., Ivano-Frankivsk, Vovchynets, 11.08.2010, 1 ○ (O. Varga); Rybne, 48.939894 N, 24.582188 E, MT, 26.05-16.06.2019, 1 o (V. Shparyk); Nadvirna Distr., Kukul, 12.08.1994, 2 ᄋ (V. Ermolenko); ZAK: Chorna Gora, MT, 20.05-7.06.2018, 1 ф; Kvasy, 29.06.2014; 1 @; MT, 15.07-10.08.2014, 2 ○; Svydovets, 27.09.2011, 2 ᄋ; Sheshul, 16-18.06.2012, 3 ○; Marmarosy, 6-9.08.2012, 1 ○ (O. Varga); Rakhiv,

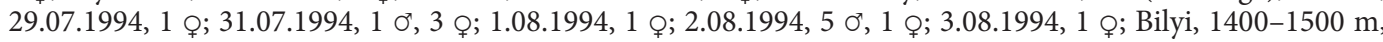
5.08.1994, 1 ९; 6.08.1994, 4 ф; 7.08.1994, 3 ○ (A. Kotenko).

\section{Distribution. Holarctic and Oriental Regions; first record for Ukraine.}

\section{Syrphophilus bizonarius (Gravenhorst, 1829) (fig. 4, A)}

Material examined. Ukraine: IF: Mochary, 17.05.2009, 1 \%; 6.05.2010, 1 \%; 25.05.2010, 1 o;

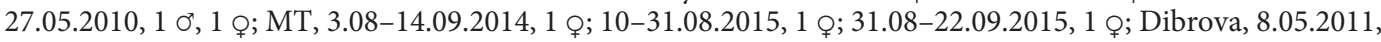
1 ơ (O. Varga); Nadvirna Distr., Chornogora, m. Pozhezhevska, 19.07.1972, 1 o; 20.07.1972, 1 \%; Chornogora, m. Dantsizh, 20.07.1972, 3 ơ; (V. Tolkanitz); ZAK: Kvasy, 23.08.2009, 1 ơ; 7.07.2012; 1 o; Sheshul, 8.06.2013, 1 ९; MT, 5-29.06.2014, 5 ᄋ; (O. Varga); 23.07.1972, 2 ơ (V. Tolkanitz); Rakhiv, 29.07.1994, 1 ९; 31.07.1994, 3 o; 3.08.1994, 2 o; 4.08.1994, 2 ơ; 13.08.1994, 1 ᄋ (A. Kotenko); Bogdan, Golovach, 28.07.1972, 5 ơ, 1 ᄋ; 29.07.1972, 1 O', 2 @; Svalyava Distr., 3 km W of Polyana, 12.07.1986, 3 \& (V. Tolkanitz); Khust Distr., Khust, 15.08.1963, 1 ○; 26.08.1963, 2 ○'.

Distribution. Holarctic and Oriental Regions; Ukraine (Klopfstein, 2014). 


\section{Syrphophilus scabriculus (Holmgren, 1858)}

Material examined. Ukraine: ZAK: Kvasy, 27.05.2011, 1 ф (O. Varga).

Distribution. Holarctic; first record for Ukraine.

\section{Syrphophilus tricinctorius (Thunberg, 1822)}

Material examined. Ukraine: IF: Mochary, 3.05.2011, 1 ơ, 3 o; 23.09.2010, 1 ○; 24.04.2010, 1 o; 4.09.2011, 1 ơ; 1.05.2013, 1 ○', 1 ○; MT, 10-29.04.2014, 5 o; 28.04-17.05.2014, 1 ○; Dibrova, 14.05.2011, 1 o'; 16.09.2011, 1 ○; 10-11.05.2012, 1 ○; 24.07.2012, 3 o'; 25.05.2013, 1 ơ; 8.10.2013, 3 ○', 15 o; 27.04.2014, 1 o'; Zhbyr, 13.09.2011, 3 ơ, 1 ᄋ; 29.09.2012, 2 ơ; Gorgany 1, 20-22.05.2012, 1 \%; 4.06.2012, 1 ơ; 8-9.06.2012, 2 ơ; Tysmenytsya Distr., Ivano-Frankivsk, Vovchynets, 11.09.2011 3 \%; Elmy, 8.06.2013, 3 ơ (O. Varga); Chornogora, 1300-1400 m, 13.07.1979, 1 \& (V. Ermolenko); ZAK: Kvasy, 5.06.2010, 2 ᄋ; 27.05.2011, 1 ᄋ; MT, 13-29.05.2015, 1 ф; Svydovets, 27.09.2011, 2 ф; Sheshul, 16-18.06.2012, 3 ơ (O. Varga).

\section{Distribution. Holarctic and Oriental Regions; first record for Ukraine.}

\section{Tymmophorus obscuripes (Holmgren, 1858) (fig. 4, B)}

Material examined. Ukraine: IF: Gorgany 1, 5.09.2009, 1 ○; 17-19.08.2011, 1 o'; Gorgany 2, 1700 m, 14.07.2012, 1 ᄋ (O. Varga); Nadvirna Distr., Kukul, 25.07.1972, 1 ○; Chornogora, m. Dantsizh, 20.07.1972, 1 ᄋ (V. Tolkanitz); ZAK: Kvasy, MT, 22.05-8.06.2015, 1 ᄋ; Chornogora, Dzhordzheva Preluka, 3 km NE of Kvasy, 48.173893 N, 24.311608 E, 1050 m, beech forest, 15.06.2012, 1 \&; Chornogora, m. Petros, 1900-1950 m, 17.06.2012, 1 申 (O. Varga); Sheshul, 23.07.1972, 1 (V. Tolkanitz); Rakhiv, 2.08.1994, 1 @; Chornogora, m. Goverla, 8.08.1994,



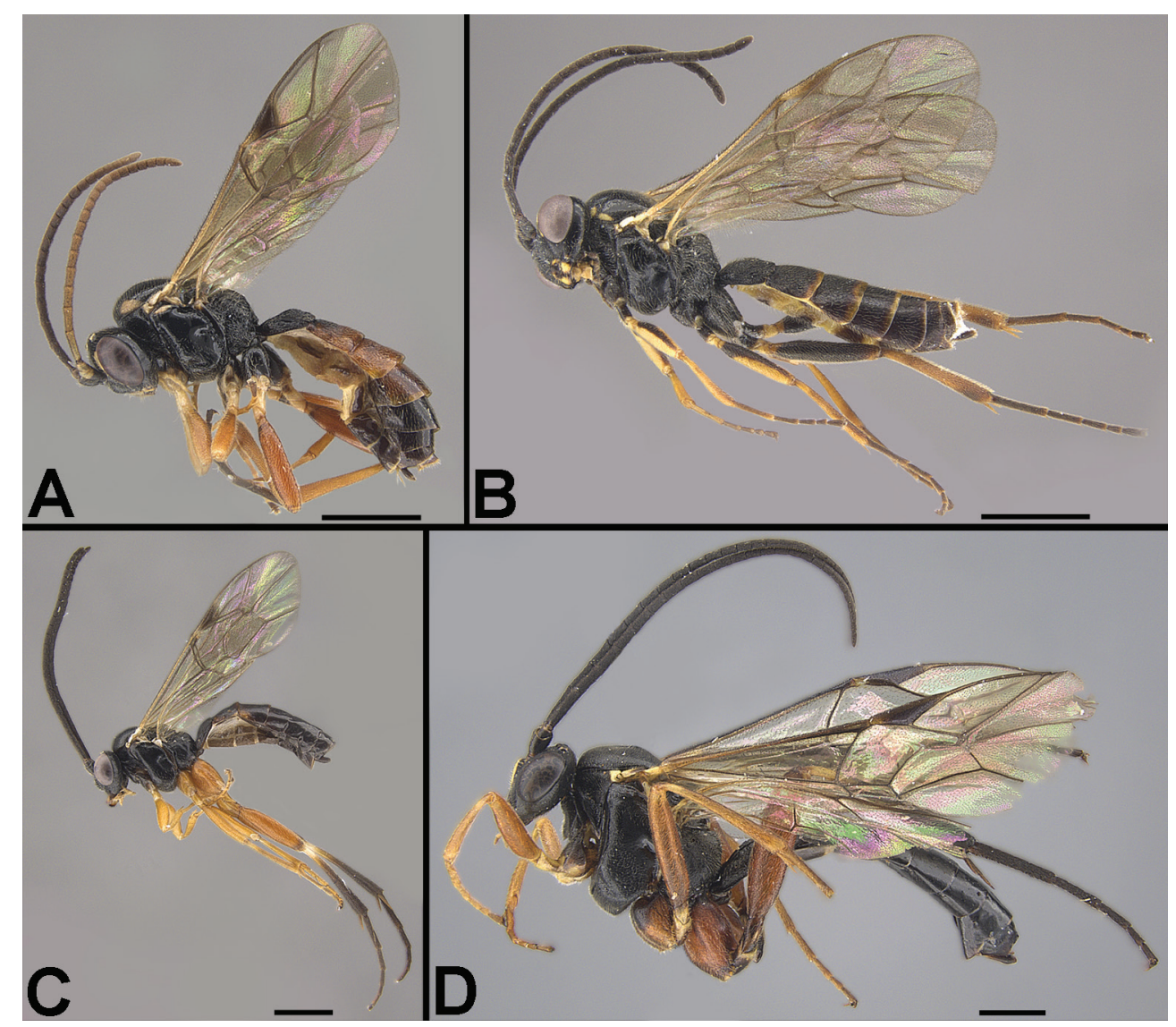

Fig. 4. The Carpathian Diplazontinae, female habitus (lateral view). A - Syrphophylus bizonarius; B - Tymmophorus obscuripes; C - Woldstedtius biguttatus; D - W. merkli. Scale bar $=1 \mathrm{~mm}$. 


\section{Distribution. Holarctic; first record for Ukraine.}

Woldstedtius biguttatus (Gravenhorst, 1829) (fig. 4, C)

Material examined. Ukraine: IF: Mochary, MT, 14-30.06.2015, 1 ○; Dibrova, 8.05.2011, 1 ơ, 1 ᄋ; Elmy, 17.07.2009, 1 \& (O. Varga); Kukul, 25.07.1972, 1 \& (V. Tolkanitz); Tysmenytsya Distr., Rybne, 48.939894 N, 24.582188 E, MT, 26.05-16.06.2019, 1 ᄋ (V. Shparyk); ZAK: Chorna Gora, MT, 12.04-2.05.2018, 1 o; Kvasy, 5.06.2010; 1 o; MT, 10.08-1.09.2014, 1 o (O. Varga); Sheshul, 23.07.1972, 1 ơ, 2 o; 9.08 .19943 o’; Rakhiv, 29.07.1994, 3 o; 31.07.1994, 5 o; 1.08.1994, 2 ơ, 1 o; 2.08.1994, 2 o; 4.08.1994, 3 o; Bilyi, $1400-$ 1500 m, 5.08.1994, 2 ơ, 2 ○; 6.08.1994, 2 ơ, 2 o; 10.08.1994, 2 ○ (A. Kotenko); Bogdan, Golovach, 28.07.1972, 2 ○; 29.07.1972, 1 ६; Svalyava Distr., Polyana, 28.06.1986, 1 \& (V. Tolkanitz).

\section{Distribution. Palaearctic; first record for Ukraine.}

\section{Woldstedtius citropectoralis (Schmiedeknecht, 1926)}

Material examined. Ukraine: IF: Dibrova, 8.10.2013, 1 ф; 10-11.05.2012, 1 ф; Gorgany 3, MT, 2-20.07.2014, 1 ᄋ; Gorgany 4, MT, 20.07-8.08.2014, 1 ᄋ; ZAK: Chorna Gora, MT, 2-20.05.2018, 1 ᄋ (O. Varga); Rakhiv, 2.08.1994, 1 ( (A. Kotenko).

\section{Distribution. Holarctic; first record for Ukraine.}

\section{Woldstedtius flavolineatus (Gravenhorst, 1829)}

Material examined. Ukraine: IF: Mochary, 17.05.2009, 1 ᄋ; Gorgany 1, 1.07.2012, 1 o; Gorgany 3, 20.06.2013, 4 o; Elmy, 20.07.2009, 1 ơ; Chornogora, 12.07.1979, 1 ơ; Chornogora, m. Pozhezhevska, 1000 m, 26.06.1975, 1 o'; 15.07.1979, 1 ơ, 1 ᄋ (V. Ermolenko); ZAK: Rakhiv, 31.07.1994, 1 ơ, 3 ᄋ; 1.08.1994, 1 ᄋ; 2.08.1994, 5 ơ, 6 \%; Chornogora, m. Goverla, SW slope, 1800 m, 8.08.1994, 1 o; Bilyi, 1500-1600 m, 5.08.1994, 1 o; 8.08.1994, 2 ○; 10.08.1994, 3 o; Uzhgorod Distr., Kamyanytsya, 4.10.1980, 1 ○’ (A. Kotenko); Khust Distr., Khust, 21.08.1963, 1 o".

\section{Distribution. Holarctic, Oriental, Oceanic, and Neotropical Regions; first record for Ukraine.}

Woldstedtius merkli Vas, 2016 (fig. 4, D)

Material examined. Ukraine: IF: Mochary, 19.06.2010, 1 \& (O. Varga).

\section{Distribution. Hungary (Vas, 2016); first record for Ukraine.}

I am deeply grateful to all collectors for provided materials for study; Brian L Fisher (California Academy of Sciences, Department of Entomology, San Francisco) for access to Leica Z16 APO microscope equipped with Leica DFC 450 camera temporarily provided for SIZK; Seraina Klopfstein (University of Bern, Institute of Ecology and Evolution, Bern) for help with identification of some species; Filippo Di Giovanni (University of Pisa, Deptartment of Agriculture, Food and Environment, Pisa) and Alexey Reshchikov (Institute of Eastern-Himalaya Biodiversity Research, Dali University, Yunnan) for valuable suggestions on the draft of the manuscript. The study was partly supported by the Rufford Small Grant for Nature Conservation, Mohamed bin Zayed Species Conservation Grant and grant of NAS of Ukraine for Young Research Groups (registration number 28/02-2020 from 16.01.2020).

\section{References}

Besser, W. V. 1835. Über die Ichneumonen Volhyniens. Bulletin de la Société Imperiale des Naturalistes de Moscou, 8, 171-176.

Klopfstein, S. 2014. Revision of the Western Palaearctic Diplazontinae (Hymenoptera, Ichneumonidae). Zootaxa, 3801 (1), 001-143.

Manukyan, A. R. 1988. Review of the genera Sussaba Cameron and Xestopelta Dasch (Hymenoptera, Ichneumonidae) of the USSR fauna. Proceedings of the Zoological Institute, Leningrad, 175, 44-54 [In Russian].

Meyer, N. F. 1936. Tables systématiques des Hyménoptères parasites (Fam. Ichneumonidae) de l'URSS et des pays limitrophes. Vol. 6. Tryphoninae. Leningrad, Akademia Nauk SSSR Press, 1-356 [In Russian]. 
Popov, M. G. 1949. Study on vegetation and flora of the Carpathians. Moskow, 1-454 [In Russian].

Rotheray, G. E. 1981. Host searching and oviposition behaviour of some parasitoids of aphidophagous Syrphidae. Ecological Entomology, 6, 79-87.

Rotheray, G. E. 1984. Host relations, life cycles and multiparasitism in some parasitoids of aphidophagous Syrphidae (Diptera). Ecological Entomology, 9 (3), 303-310.

Rotheray, G. E. 1990. A new species of Bioblapsis (Hymenoptera: Ichneumonidae) from Scotland parasitising a mycophagous hoverfly, Cheilosia longula (Diptera: Syrphidae). Entomologica Scandinavica, 21, 277-280.

Varga, A. 2014 a. A review of the subfamily Rhyssinae (Hymenoptera, Ichneumonidae) from the Ukrainian Carpathians. Vestnik Zoologii, 48 (1), 27-34.

Varga, O. 2014 b. New data on the genus Dolichomitus (Hymenoptera, Ichneumonidae, Pimplinae) in the Ukrainian Carpathians. Vestnik Zoologii, 48 (4), 325-332.

Varga, O. 2015. A review of the subfamily Poemeniinae Narayanan \& Lai, 1953 (Hymenoptera, Ichneumonidae) from Carpathians. Journal of Insect Biodiversity, 3 (7), 1-14.

Varga, O. 2018 A review of the tribe Delomeristini (Hymenoptera, Ichneumonidae, Pimplinae) in the Ukrainian Carpathians. Vestnik Zoologii, 52 (3), 235-240.

Vas, Z. 2016. Woldstedtius merkli sp. n. from Hungary (Hymenoptera: Ichneumonidae). Folia Entomologica Hungarica, 77, 57-65.

Yu, D. S., van Achterberg, K., Horstmann, K. 2012. World Ichneumonoidea 2011. Taxonomy, Biology, Morphology and Distribution. (Flash drive version).

Received 21 April 2020

Accepted 24 April 2020 